\title{
Planomonospora: a Metabolomics Perspective on an Underexplored Actinobacteria Genus
}

\author{
Mitja M. Zdouc, ${ }^{*,+, \ddagger}$ Marianna lorio, ${ }^{\dagger}$ Sonia I. Maffioli, ${ }^{\dagger}$ Max Crüsemann, \\ Stefano Donadio, ${ }^{\dagger}$ and Margherita Sosio*, ${ }^{*}$ \\ $\dagger$ Naicons Srl., Viale Ortles 22/4, 20141 Milano \\ $\ddagger$ University of Amsterdam, SILS, Science Park 904, 1098 XH Amsterdam, The \\ Netherlands. \\ \Institut für Pharmazeutische Biologie, Rheinische Friedrich-Wilhelms-Universität, \\ Nußallee 6, 53115 Bonn, Germany \\ E-mail: zdoucmm@gmail.com; msosio@naicons.com
}

\begin{abstract}
:
Despite an excellent track record, microbial drug discovery suffers from high rates of re-discovery. Better workflows for the rapid investigation of complex extracts are needed to increase throughput and allow early prioritization of samples. In addition, systematic characterization of poorly explored strains is seldomly performed. Here, we report a metabolomic study of 72 isolates belonging to the rare actinomycete genus Planomonospora, using a workflow of open access tools to investigate its secondary metabolites. The results reveal a correlation of chemical diversity and strain phylogeny, with classes of metabolites exclusive to certain phylogroups. We were able to identify previously reported Planomonospora metabolites, including the ureylene-containing oligopeptide antipain, the thiopeptide siomycin including new congeners and the ribosomally
\end{abstract}


synthesized peptides sphaericin and lantibiotic 97518. In addition, we found that Planomonospora strains can produce the siderophore desferrioxamine or a salinichelin-like peptide. Analysis of the genomes of three newly sequenced strains led to the detection of 47 gene cluster families, of which several were connected to products found by LC-MS/MS profiling. This study demonstrates the value of metabolomic studies to investigate poorly explored taxa and provides a first picture of the biosynthetic capabilities of the genus Planomonospora. 


\section{Introduction:}

Natural products are excellent sources for bioactive scaffolds. Over the last four decades, $66 \%$ of approved small-molecule drugs were actual natural products or at least inspired from such. ${ }^{1}$ This is an impressive track record, considering the general withdrawal of industrial activity from the field. ${ }^{2}$ One reason for this disinterest has been the frequent rediscovery of known molecules in activity-based screenings, especially in microbe-derived extracts. ${ }^{3}$ However, the focus on bioactivity as selection criterion provides a biased perspective on a small portion of the chemical diversity microbes are capable to produce. Despite decades of research, the majority of secondary metabolites remain "metabolomic dark matter", 4 with high probability of structural novelty ${ }^{5}$ and novel bioactive scaffolds. ${ }^{6}$ Still, streamlined approaches for strain prioritization and workflow optimization are needed to render drug discovery from microbial sources a cost-effective endeavor. ${ }^{5}$

Recent advances in genome mining have enabled researchers to investigate the biosynthetic potential of bacteria in silico, with minimal wet lab work. ${ }^{7}$ Tools such as antiSMASH ${ }^{8}$ allow to mine genomes for biosynthetic gene clusters (BGC), while BGC repositories such as $\mathrm{MIBiG}^{9}$ aid in the evaluation of BGC novelty. In addition, advances in (tandem) mass spectrometry and the introduction of molecular networking, ${ }^{10}$ the tandem mass $\left(\mathrm{MS}^{2}\right)$ based grouping of molecules by structural relatedness, has made untargeted metabolomics broadly available, ${ }^{11}$ while public databases in the likes of GNPS ${ }^{12}$ and the Natural Product Atlas ${ }^{13}$ facilitate metabolite annotation. These methods allow to rationalize resources and quickly prioritize strains or metabolites for further investigations. Earlier studies on bacterial genera, such as the actinobacteria Salinispora ${ }^{14-16}$ and Nocardia, ${ }^{17}$ the myxobacterium Myxococcus ${ }^{18}$ and the gamma-proteobacterium Pseudoalteromonas ${ }^{19}$ have demonstrated distinct chemical profiles and shown correlations between taxonomic and metabolomic diversity.

We are particularly interested in exploring the metabolic capabilities of "rare" genera of actinomycetes present within the Naicons collection, which comprises approximately 45,000 actinomycete strains of diverse origin, isolated between 1960 and $2005 .{ }^{20}$ One such genus 
is Planomonospora. Originally described by researchers from Lepetit (the predecessor company of Naicons) in $1967,{ }^{21}$ so far, just six species, two sub-species and four unclassified strains can be found in public collections or databases. Only few molecules have been described as produced by representatives of this genus: the thiopeptides thiostrepton ${ }^{22}$ and sporangiomycin, ${ }^{23}$ the latter identical to siomycin, produced also by Streptomyces sioyaen$\operatorname{sis}^{24}$ (and henceforth referred to as such); lantibiotic 97518, also known as planosporicin, ${ }^{25,26}$ a member of a family of class I lantipeptides produced by many actinobacterial genera; ${ }^{27}$ the lassopeptide sphaericin; ${ }^{28}$ and the ureylene-containing oligopeptide antipain, ${ }^{29}$ which is also produced by Streptomyces. ${ }^{30}$ Here, we aim to assess the capability of Planomonospora strains to produce secondary metabolites, using a pipeline of freely available tools for metabolome and genome mining. The modular nature of our workflow facilitates fast and flexible processing of data, allowing to quickly prioritize strains and/or metabolites for further investigation (see Figure 1). The investigation was carried out on 72 strains from the Naicons collection and was complemented by selected genomic analyses. This study gives unprecedented insight into the rare genus Planomonospora, correlating metabolites to their putative biosynthetic gene clusters and making way for targeted isolation efforts.

\section{Results and Discussion:}

\section{Determination of Phylogenetic Affiliation:}

From the over 350 Planomonospora entries listed in the Naicons collection, we selected 72 strains confirmed by $16 \mathrm{~S}$ rRNA gene sequencing as belonging to the genus Planomonospora. As far as information was available, all strains were isolated from soil samples, with the majority originating from central Africa and the Mediterranean region (see Figures S1 and $\mathrm{S} 2)$. Overall, the 72 strains yielded 35 unique $16 \mathrm{~S}$ rRNA sequences, 31 of which were not reported previously (see Figures S1 and S2). The resulting phylogenetic tree (see Figure 2) was found to be in agreement with previous studies ${ }^{31,32}$ and showed three phylogroups 


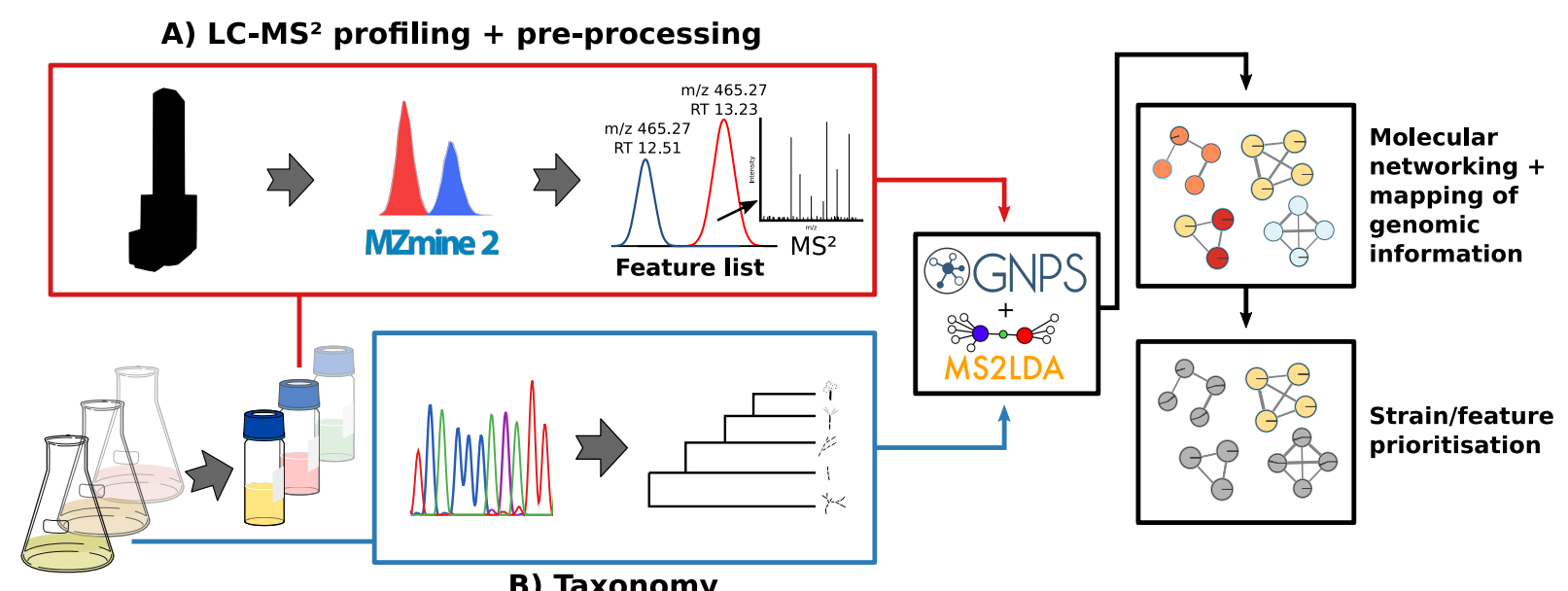

B) Taxonomy

Figure 1. Workflow: Strains are cultivated, extracts are prepared and analyzed. (A) Data dependent acquisition (DDA) mode on an ESI-HR-LCMS-MS-instrument. Data are preprocessed with the MZmine2 software, yielding a list of features, which are then analyzed by GNPS feature-based molecular networking, with consecutive MS2LDA curation. (B) Taxonomy of the strains is established by $16 \mathrm{~S}$ rRNA-sequencing. Strains/features are prioritized for consecutive targeted isolation.

with a relevant number of representatives: phylogroup "C" includes 13 strains (9 of them from Naicons collection) and 9 distinct 16S rRNA sequences; phylogroup "A2" includes 12 strains (11 of them from Naicons collection) and 8 distinct 16S rRNA sequences; and the most populated phylogroup "S" includes 46 strains (43 of them from Naicons collection) and 13 distinct $16 \mathrm{~S}$ rRNA sequences. In addition, the phylogenetic analysis yielded three poorly represented phylogroups: "V1", which includes Planomonospora venezuelensis JCM3167 and Naicons strain ID43178, with identical 16S rRNA sequences; the somehow related "V2" group, with six distinct Naicons isolates with identical sequences; and "A1", with just two Naicons strains with identical sequences. All phylogroups contained sequences of previously described Planomonospora species, except "V2" and "A1". Given the extent of sequence distance from validly described species (see Figures S1 and S2), many of the Naicons strains likely represent new species within this genus. In the following analyses, we consider "V1" as a phylogroup, even though it contains only one strain. 


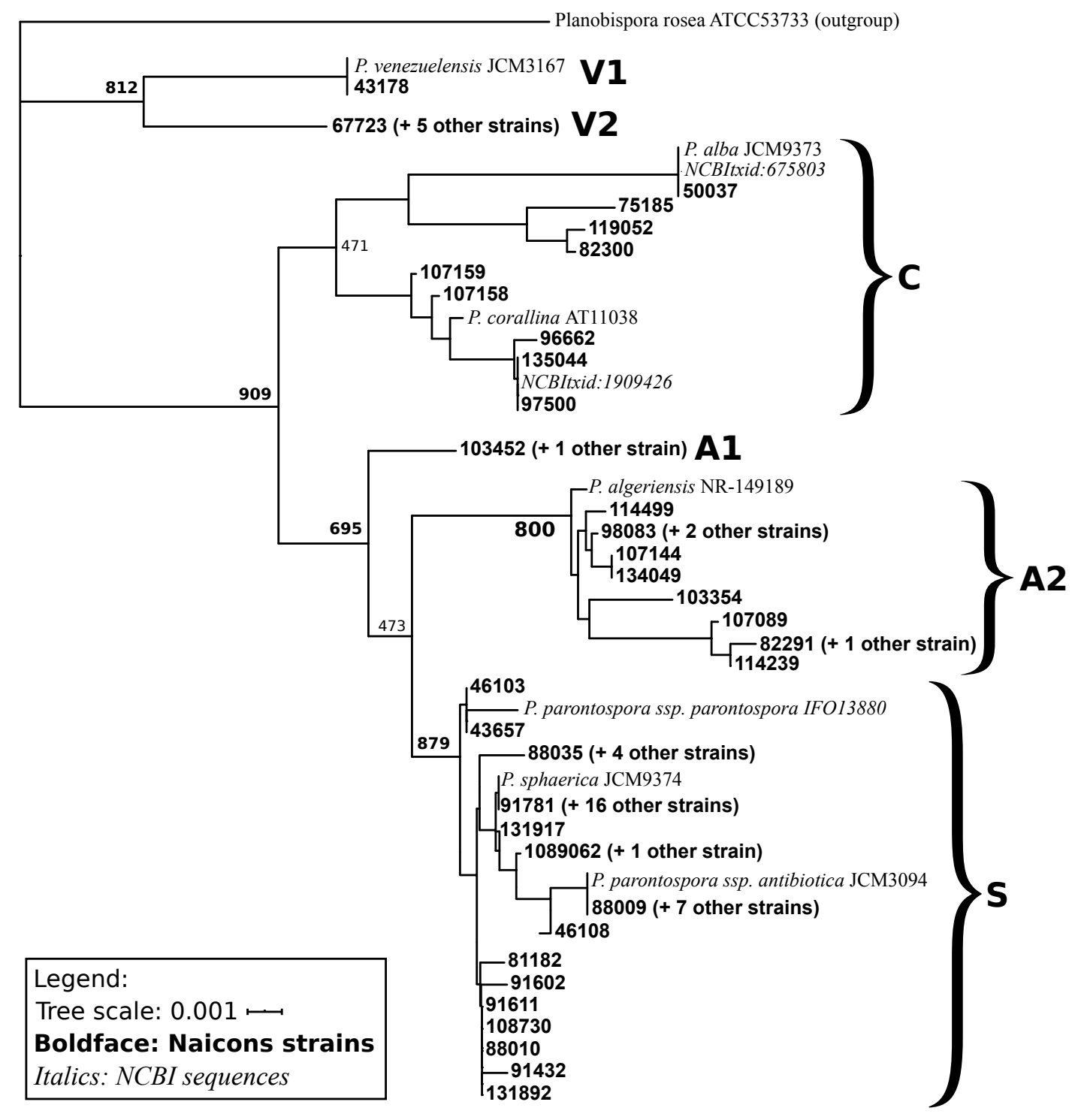

Figure 2. 16S rRNA-based phylogenetic tree of Planomonospora(P.)-strains. Naicons strains are represented by their ID numbers in boldface. Type strains and two unclassified strains with complete 16S sequences are indicated in italics. Naicons strains with identical sequences are represented by a single ID number, with the number of additional strains in parentheses (details in Figures S1 and S2). Bootstrap values (1000 times resampled) higher than $60 \%$ are indicated in bold type. Planobispora rosea ATCC53733 was used as outgroup.

\section{Cultivation, Extraction and Molecular Network Analysis:}

To find appropriate cultivation conditions for the Planomonospora strains, we first explored the behavior of a selected number of isolates under a variety of different conditions, including three solid and six liquid media. Four liquid media (AF, R3, MC1 and AF2, see Experimental 
Procedures) afforded the highest metabolic diversity (data not shown) and were used in the following analyses.

Cultivation of the 72 strains in the four media and preparation of two extracts per culture yielded 576 samples. To expedite analysis, the two extract from each culture were combined, yielding 288 samples, two of which were removed due to cross-contamination. The remaining 286 samples were analyzed by high resolution ESI-LC-MS/MS in data dependent acquisition mode. The LC-MS/MS data were subjected to a workflow consisting of several steps (see Figure 1): files were (i) preprocessed with the feature finding tool MZmine2 to take into account chromatography well-resolved isomers as well as to correct for $\mathrm{m} / z$ and retention time drift; ${ }^{33,34}$ (ii) analyzed using the feature-based molecular networking workflow of GNPS; ${ }^{12,35}$ and (iii) visualized using the program Cytoscape ${ }^{36}$ (see Figure S3).

As seen in Figure 3, the molecular network contains 1492 features, with media components, background impurities (from the extraction process) as well as features with less than $m / z 300.0$ removed beforehand (to exclude primary metabolism). Of those 1492 features, 447 (30\%) were organized in 60 molecular families. The remaining 1045 features were singletons. The molecular network is a visual representation of the chemistry detected by mass spectrometry. The network is comprised of sub-networks (called molecular families), which are in turn made up of features (=nodes) and edges. Each feature is a non-redundant representation of a detected parent ion over all samples, with a distinct mass-to-charge-ratio $(m / z)$, retention time $(\mathrm{RT})$ and corresponding tandem mass $\left(\mathrm{MS}^{2}\right)$ fragmentation spectra (see Figure S3). For example, soyasaponin I, a soymeal-related medium component, would be represented as a single feature with a specific $m / z$, RT and $\mathrm{MS}^{2}$ spectrum, even though it is detected in almost all samples. Still, the number of features is not equivalent to the number of metabolites, since the same metabolite can be detected as different adducts (and thus features) by ESI mass spectrometry (e.g. $[\mathrm{M}+\mathrm{H}]^{+},[\mathrm{M}+2 \mathrm{H}]^{2+},[\mathrm{M}-2 \mathrm{H}+\mathrm{Fe}]^{+},[\mathrm{M}+\mathrm{Na}]^{+}$ ...). Features can be further connected by edges, which represent the pairwise similarity between two $\mathrm{MS}^{2}$ fragmentation spectra. Since similar $\mathrm{MS}^{2}$ fragmentations indicate a similar 
chemical structure of the parent ions, connected features are considered to be structurally related. ${ }^{10}$ Molecular families are therefore topological representations of chemical relatedness. Features can also be singletons, if they have sufficiently unique $\mathrm{MS}^{2}$ spectra not to cluster with any other feature. For the following investigation, all 1492 features were taken into account, independent of their topological organization (singleton or member of a molecular family).

To explore trends in feature distribution, sample metadata, such as producing strain, cultivation medium and phylogroup affiliation, can be mapped onto the molecular network, which supports intuitive assessment and helps in data organization. An example is shown in Figure 3, where node color correlates to phylogroups. After this sort of visualization, we systematically explored occurrence of features according to strain, phylogroup and medium, as explained below.

We first investigated how features varied within and between phylogroups. A recent study on myxobacteria demonstrated strong correlation between taxonomic and secondary metabolite diversity, i.e. metabolite profiles showed high taxonomic specificity. ${ }^{37}$ This raised the question whether this applied to Planomonospora as well. Indeed, only $1 \%$ of features were detected in members of all phylogroups, as shown by the Venn diagram in Figure 4A. The vast majority (74\%) of the 1492 features were phylogroup-specific, meaning that they were not detected in samples derived from strains of a different phylogroup. The number of specific features was especially high for phylogroup "C". Despite counting only 9 strains, $31 \%$ of all detected features were exclusive to its members. This is consistent with the phylogenetic tree of Figure 2, which indicates that phylogroup " $\mathrm{C}$ " is more divergent from the other well-represented phylogroups "A" and "S". In contrast, the number of phylogroupspecific features was relatively low in groups "A1" and "A2", suggesting that the separation into two phylogroups might be an artifact due to the existence of only one 16S rRNA sequence in phylogroup A1. Overall, the results suggest that secondary metabolite production in Planomonospora ssp. is a phylogroup-defining trait. 
bioRxiv preprint doi: https://doi.org/10.1101/2020.07.19.210815; this version posted July 19, 2020. The copyright holder for this preprint (which was not certified by peer review) is the author/funder, who has granted bioRxiv a license to display the preprint in perpetuity. It is made available under aCC-BY-NC-ND 4.0 International license.

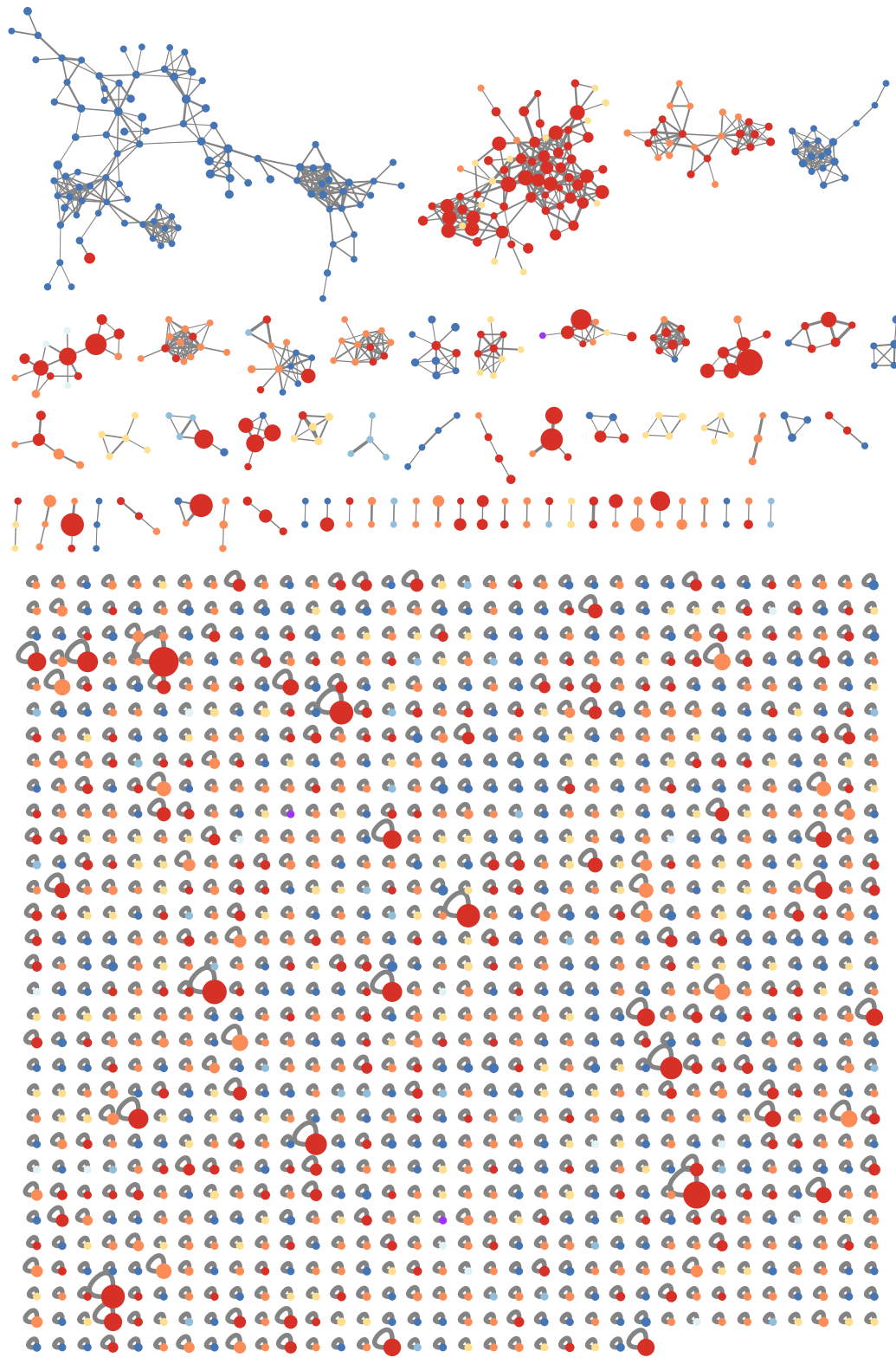

\section{1 \\ Node size $=$ number of strains}
$0 \geq 2$ phylogroups
"S"-phylogroup
"C"-phylogroup
"V2"-phylogroup
"A2"-phylogroup
"A1"-phylogroup
"V1"-phylogroup

Figure 3. Complete molecular network of 286 Planomonospora extracts, encompassing 1492 features (=nodes). 447 features were organized in 60 molecular families. Node size correlates to the number of contributing strains, while the colors give the contributing phylogroup(s). 
We also investigated the distribution of features at a strain level. Each bar in Figure 4B represents a single strain, with the number of detected features indicated on the y-axis. It can be seen that this number varies greatly among strains, with some "talented" strains standing out in terms of both total features and strain-specific features. Phylogroup "C" again, and, to a lesser extent, "V2", were enriched in such strains. This is particularly relevant for phylogroup "V2", for which all six strains shared an identical 16S rRNA sequence. In total, $36 \%$ of features were strain-specific, indicating that also for the genus Planomonospora, secondary metabolites tend to be strain-specific.

We were also interested in the effects of the different cultivation media on metabolite profiles of strains. Since all 72 strains were cultivated in the same four media, feature distributions could be evaluated (see Figure 5). Overall, 57\% of features were mediumspecific. Media MC1 and R3, with 26 and 18\% of exclusive features, respectively, were the biggest contributors. Further, these two media covered $85 \%$ of all detected features. In contrast, just $9 \%$ of features were found in all media. Similar observation were made in a study of 26 marine Streptomyces strains, in which $71 \%$ of detected ions were mediumspecific and just $7 \%$ common to all 3 conditions. ${ }^{14}$ Even though complete metabolite coverage remains elusive, our results suggest that two media should cover most of the metabolites produced by different Planomonospora strains belonging to different phylogroups.

\section{Metabolite Annotation:}

Next, we started examining the actual metabolites present in the Planomonospora metabolome by first identifying known metabolites in a process termed de-replication. ${ }^{38,39}$ In addition to providing insights into the biosynthetic potential of this poorly studied genus, establishing the known metabolites can highlight features likely to be associated with novel chemistry, thus evading the pitfalls of re-investigating reported compounds. As described below, we de-replicated representatives of 11 molecular families, some of which are visualized in Figure 6. For annotation, we used both spectral matching (comparison with identified spectra 

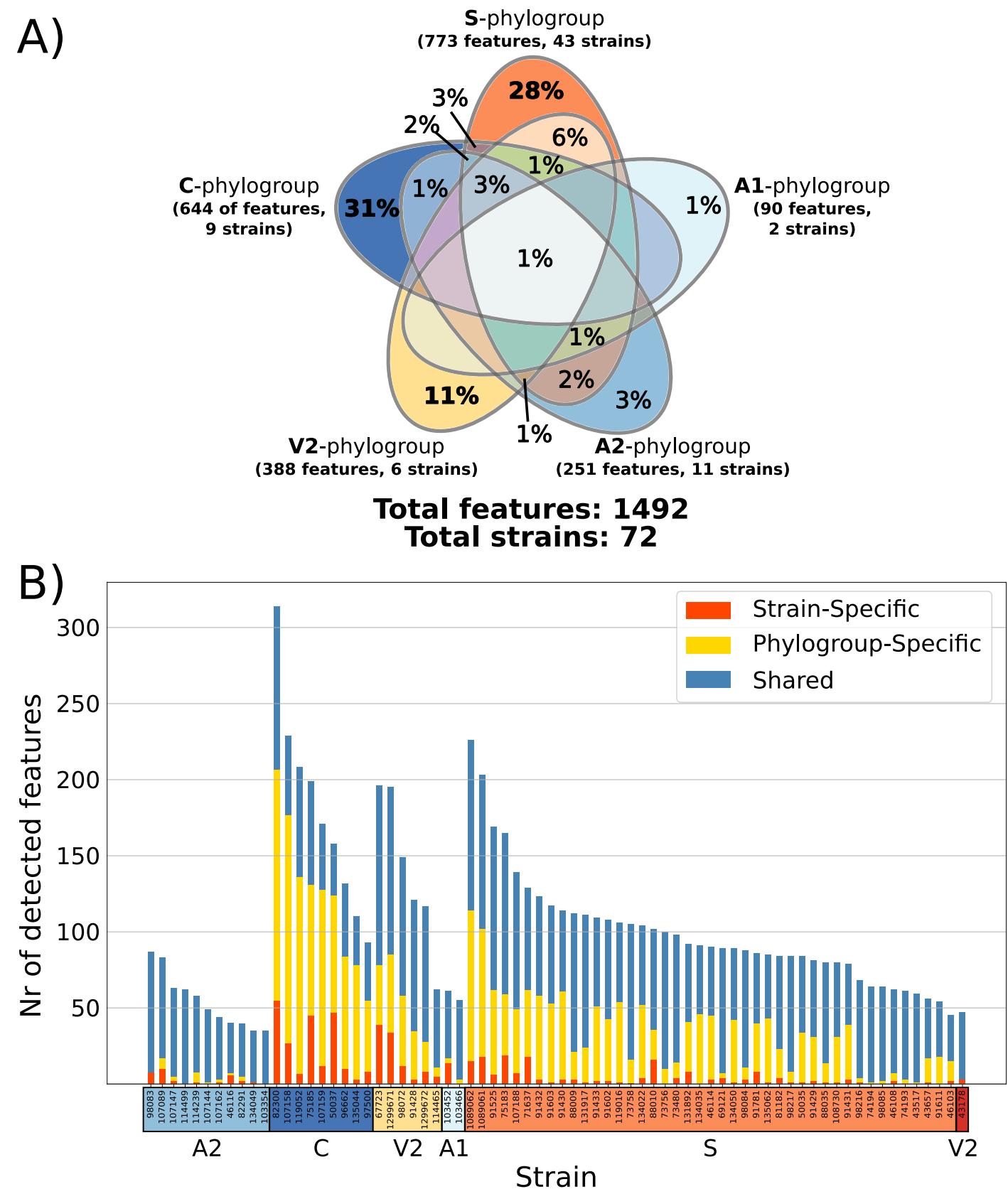

Figure 4. Distribution of 1492 features according to (A) phylogroups and (B) strains. In panel A, overlaps amounting to less than $1 \%$ are not labeled, while features detected in phylogroup "V1" were omitted from the analysis. In panel B, each bar represents a different strain. Bars are separated into strain-specific (red), phylogroup-specific (yellow, detected in at least one additional strain from the same phylogroup) and shared features (blue, detected in at least one additional strain from a different phylogroup). 


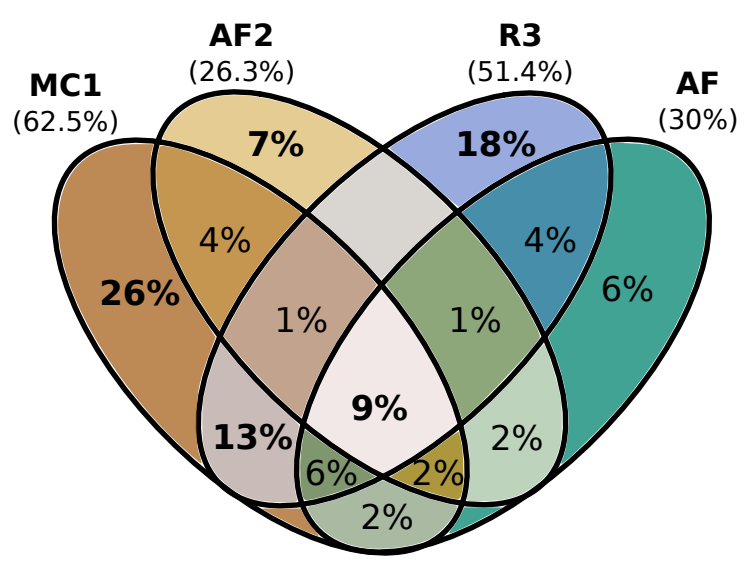

Total features: 1492

Figure 5. Visualization of the distribution of features in extracts with regard to cultivation medium. Media are MC1, AF2, R3 and AF. Overlaps amounting to less than 1\% are not labeled.

in curated databases, such as GNPS) and literature search. To increase confidence in the annotations, Chemical Analysis Working Group (CAWG) criteria $^{40}$ were applied, leading to a number of class 1 (comparison against an authentic standard) and class 2 (putatively identified molecule) annotations (for a full list, see Figure S4).

We first searched for the metabolites previously reported from Planomonospora. The lantibiotic 97518 was identified in an extract from strain ID50037 after observing a signal with $m / z$ 1097.39, which matched the $[\mathrm{M}+2 \mathrm{H}]^{2+}$ ion (representing the isotope containing 1 ${ }^{13} \mathrm{C}$ atom), and comparison of its $\mathrm{MS}^{2}$ fragmentation spectrum to the one reported in the literature confirmed this assumption (see Figures S4 and S31). ${ }^{25}$ The lassopeptide sphaericin, identified by the signal $m / z$ 2157.12, corresponding to the $[\mathrm{M}+\mathrm{H}]^{+}$ion (representing the isotope containing $1{ }^{13} \mathrm{C}$ atom) was found in the extracts of six strains. ${ }^{28}$ Again, its $\mathrm{MS}^{2}$ fragmentation spectrum matched the one reported in the literature (see Figures S4 and S32). Further, the thiopeptide siomycin $\mathrm{A}\left([\mathrm{M}+\mathrm{H}]^{+} 1648.46 \mathrm{~m} / z\right)$, along with its congeners, siomycin $\mathrm{B}\left([\mathrm{M}+\mathrm{H}]^{+} 1510.42 \mathrm{~m} / z\right)$, siomycin $\mathrm{C}\left([\mathrm{M}+2 \mathrm{H}]^{2+} 832.73 \mathrm{~m} / z\right)$ and siomycin D1 $\left([\mathrm{M}+2 \mathrm{H}]^{2+} 817.73 \mathrm{~m} / z\right)$ was detected in extracts of up to 26 strains and will be described in detail below. Finally, a signal with $m / z$ 303.18, corresponding to the $[\mathrm{M}+2 \mathrm{H}]^{2+}$ ion of 
ureylene-containing oligopeptide antipain, was detected in extracts of 11 strains and annotated by comparison to an authentic standard (see Figures S4 and S20). Several more signals belonging to ureylene-containing oligopeptides were identified: the antipain-like molecule KF 77AG6 $\left([\mathrm{M}+\mathrm{H}]^{+} 366.18 \mathrm{~m} / z\right.$, Figures $\mathrm{S} 4$ and $\left.\mathrm{S} 29\right)$ as well as chymostatin A/C $\left([\mathrm{M}+\mathrm{H}]^{+}\right.$ $608.31 \mathrm{~m} / z$, Figures $\mathrm{S} 4$ and $\mathrm{S} 24)$ along with its congeners chymostatin $\mathrm{B}\left([\mathrm{M}+\mathrm{H}]^{+} 594.30\right.$ $m / z$, Figures S4 and S25), chymostatinol A $\left([\mathrm{M}+\mathrm{H}]^{+} 596.32 \mathrm{~m} / z\right.$, Figures $\mathrm{S} 4$ and S26), chymostatinol B $\left([\mathrm{M}+\mathrm{H}]^{+} 610.33 \mathrm{~m} / z\right.$, Figures S4 and S27) and GE-20372 A/B $\left([\mathrm{M}+\mathrm{H}]^{+} 612.31\right.$ $m / z$, Figures S4 and S28). Except for thiostrepton, all previously reported Planomonospora metabolites were identified in our dataset. In addition, we were able to detect several members of the desferrioxamine family, an iron chelating siderophore commonly produced by Streptomyces. ${ }^{41}$ The signal corresponding to desferrioxamine B $[\mathrm{M}+\mathrm{H}]^{+}$ion $(561.36 \mathrm{~m} / z)$, detected in extracts of 8 strains, was annotated by comparison to a commercial standard (see Figures S4 and S30). Further, signals matching Acyl Desferrioxamin C13, C15 and C16 $\left([\mathrm{M}+\mathrm{H}]^{+} 729.54 \mathrm{~m} / z,[\mathrm{M}+\mathrm{H}]^{+} 757.58 \mathrm{~m} / z\right.$ and $[\mathrm{M}+\mathrm{H}]^{+} 771.59 \mathrm{~m} / z$, Figures $\mathrm{S} 4$ and $\mathrm{S} 21-23$, respectively) were identified by literature search. Some other putative siderophores were identified by spectral matching against the GNPS spectral library (see Figure S4): desferrioxamine $\mathrm{E}\left([\mathrm{M}+\mathrm{H}]^{+} 601.36 \mathrm{~m} / z\right)$, as well as partially described metabolites deposited as "amphiphilic ferrioxamine 7" $\left([\mathrm{M}+\mathrm{H}]^{+} 768.44 \mathrm{~m} / z\right)$, "Bisu-05" $\left([\mathrm{M}+\mathrm{H}]^{+} 345.35 \mathrm{~m} / z\right)$ and "Desf-05" ([M+H]+ $575.37 \mathrm{~m} / z)$.

Several of the de-replicated features showed strong phylogroup-specificity: the desferrioxamines were almost exclusively detected in samples from strains of phylogroup "C" (see Figure 6A), while siomycins and sphaericin were only detected in extracts derived from the "S" phylogroup strains (Figure 6C). Other metabolites were less phylogroup-specific: chymostatinol A was produced by 31 strains from phylogroups "C", "S", "V2" and "A2", as were antipain (11 strains, phylogroups "S", "V2" and "A2") or GE-20372 A/B (17 strains, phylogroups "C", "S", "V2" and "A2"). The other identified ureylene-containing oligopeptides showed a similar broad distribution. 
In total, 28 metabolites were annotated as CAWG classes 1 or 2 (see Figure S4). A summary of the presence/absence of all de-replicated features can be seen in Figure S5. Many more features in the molecular network are neighbours to annotated ones (and thus, structurally related). While a systematic investigation of all features would exceed the scope of this study, examples of special relevance will be discussed below. To get a better picture of the biosynthetic capacities of Planomonospora and to further explore the annotated metabolites, we turned to genomic analysis.

\section{Genome analysis:}

Public databases report only a single Planomonospora genome sequence, namely that of Planomonospora sphaerica JCM9374. ${ }^{42}$ Thus, we selected three representative strains for full genome sequencing: strain ID67723, as a representative of the divergent phylogroup "V2" and for its capability to produce chymostatin; strain ID82291 as the representative of a subgroup of strains in phylogroup "A2" that produced a family of modified peptides, as reported elsewhere; ${ }^{43}$ and strain ID91781, with its $16 \mathrm{~S}$ rRNA sequence identical to that of $P$. sphaerica JCM9374 and a producer of the thiopeptide siomycin. Genomes were sequenced with both Illumina HiSeq and PacBio technologies to allow hybrid assembly, providing good quality sequences with a substantially lower number of contigs that the reference genomes of P. sphaerica JCM9374 and of Planobispora rosea ATCC53733. ${ }^{44}$ The three genomes were similar to that of P. sphaerica JCM9374 and to each other in terms of GC content (from 71.5 to $72.83 \%$, see Figure 7B). Interestingly, the genome of strain ID82291 was about 9\% smaller than the other Planomonospora genomes and thus harboured a smaller number of predicted genes. antiSMASH analysis identified between 23 and 28 biosynthetic gene clusters (BGCs) in the three genomes (see Figure 7A).

A multilocus sequence analysis of the five strains of Figure 7A, along with other publicly available genome sequences of members of the Streptosporangiaceae, was performed with the web-based program autoMLST. ${ }^{45}$ Based on a concatenated alignment of 89 identified 


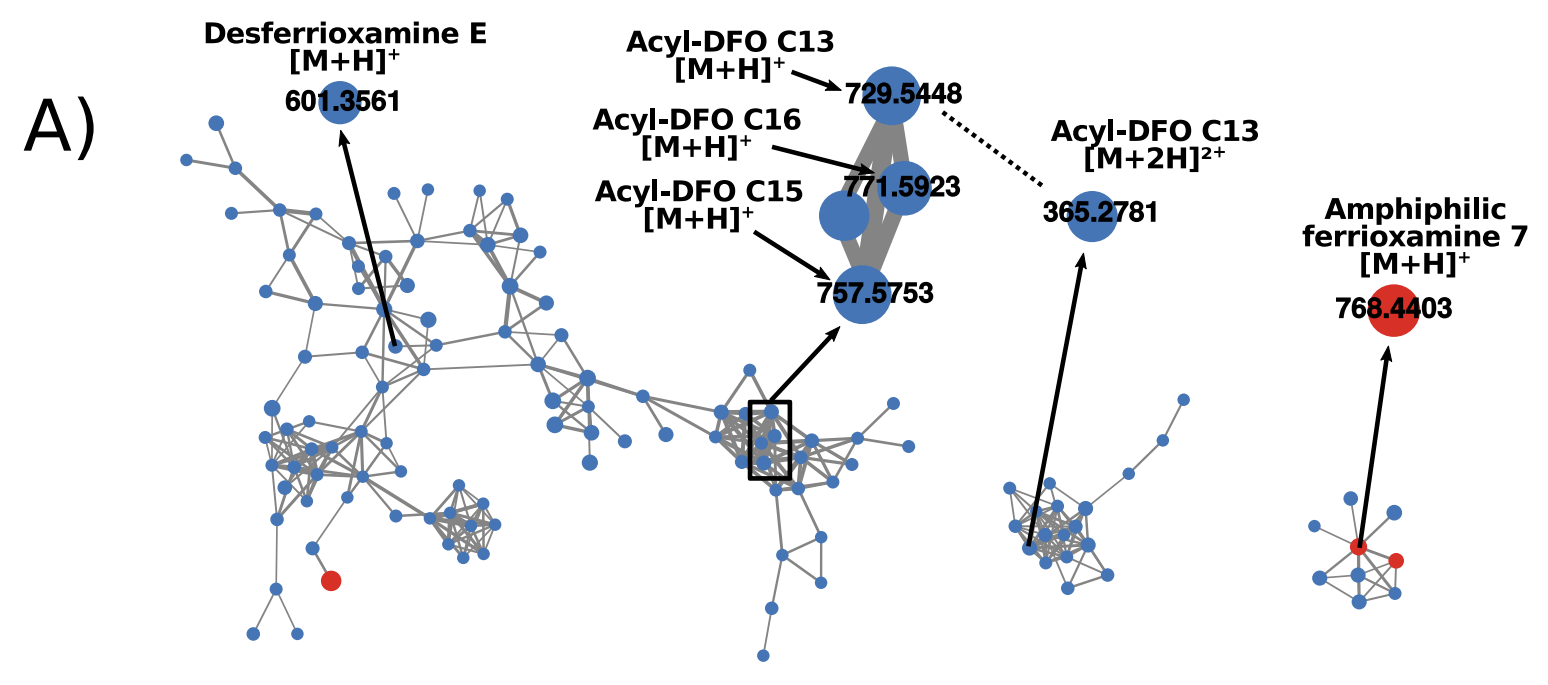

B)

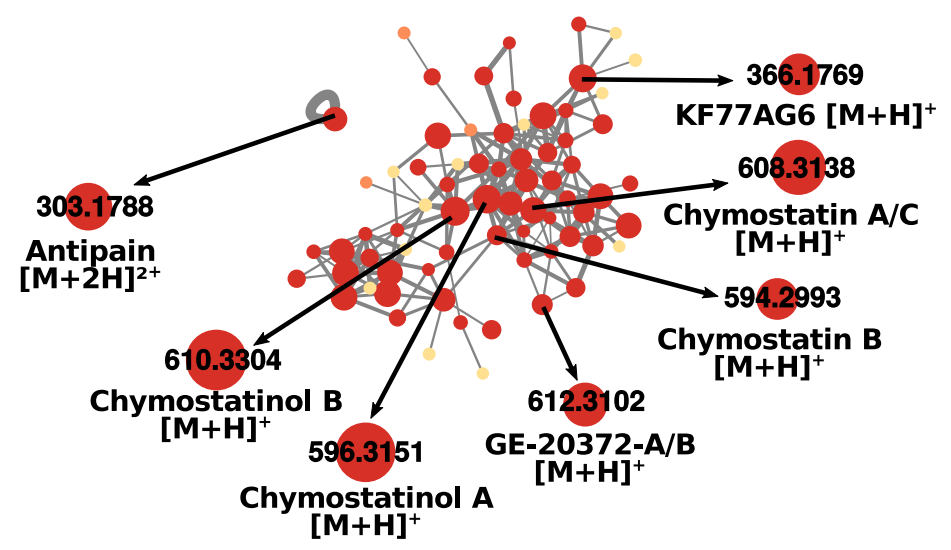

C)

$\geq 2$ phylogroups

"S"-phylogroup

"C"-phylogroup

"V2"-phylogroup

Figure 6. Visualisation of selected annotated molecular families, with features annotated manually or by GNPS spectral library search (e.g. "amphiphilic ferrioxamine 7"). (A) desferrioxamines (DFO) are mostly occurring in strains from phylogroup " $\mathrm{C}$ ". (B) chymostatin-like metabolites show no phylogroup-specificity. (C) Siomycin and sphaericin are exclusive to phylogroup "S".

housekeeping genes (see Figure S38), a tree was constructed that showed consistent with the one of Figure 2, except that strain ID91781 is now clearly distinct from Planomonospora sphaerica JCM9374 (see Figure 7B). Oddly, strain ID67723 shows closer relationship to Planobispora rosea than to the other Planomonospora strains, in contrast to the results from the 16S rRNA-based phylogeny (see Figure 2). These results warrant further studies in the taxonomy of Planomonospora and Planobispora ssp. 


\begin{tabular}{|c|c|c|c|c|c|}
\hline StrainID & $\begin{array}{l}\text { P. sphaerica } \\
\text { JCM9374 }\end{array}$ & P. ID91781 & P. ID82291 & P. ID67723 & $\begin{array}{l}\text { Planobisp, rosea } \\
\text { ATCC53733 }\end{array}$ \\
\hline Bases (Mbp) & 8.13 & 8.28 & 7.58 & 8.34 & 8.69 \\
\hline Contigs & 84 & 6 & 15 & 9 & 118 \\
\hline GC-content (\%) & 72.7 & 72.6 & 72.8 & 71.5 & 71.2 \\
\hline Genes predicted & 7224 & 7243 & 6754 & 7560 & 7851 \\
\hline $\begin{array}{l}\text { BGCs predicted } \\
\text { NCBI Accession }\end{array}$ & $\begin{array}{l}28 \\
\mathrm{NZ} B \mathrm{BDC} \times 00 \\
000000\end{array}$ & $\begin{array}{l}27 \\
\text { SAMN14970434 }\end{array}$ & $\begin{array}{l}23 \\
\text { SAMN14970435 }\end{array}$ & $\begin{array}{l}26 \\
\text { SAMN14970433 }\end{array}$ & $\begin{array}{l}27 \\
\text { NZJPMWW00 } \\
000000\end{array}$ \\
\hline
\end{tabular}
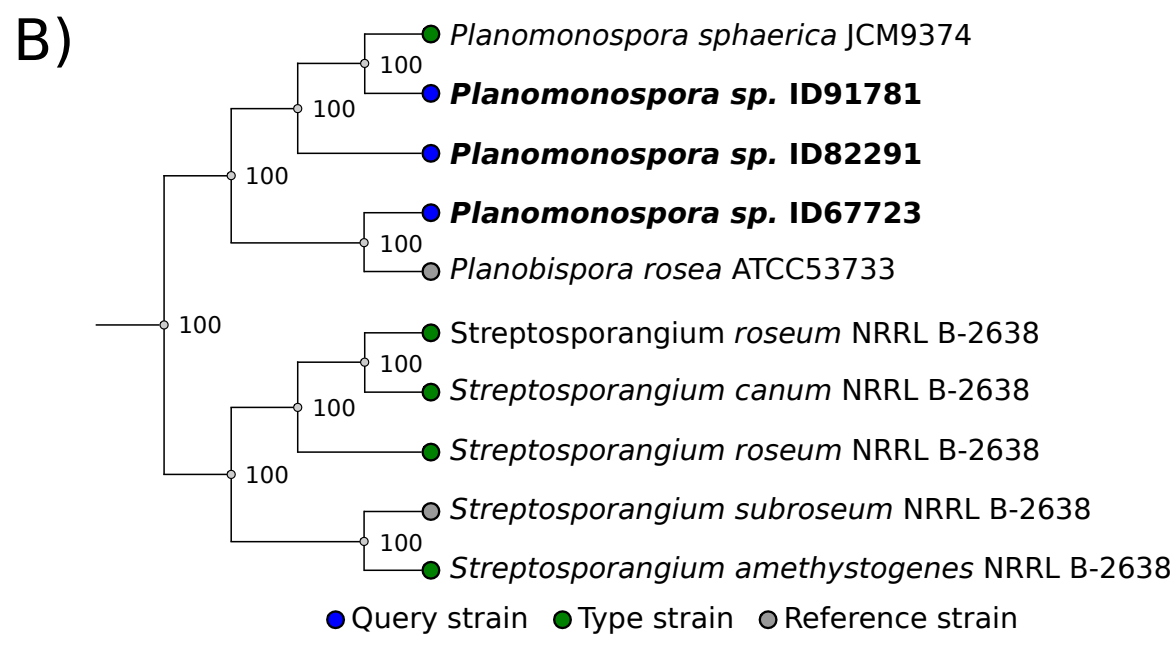

Figure 7. Overview of sequenced genomes, with comparison to reported ones. (A) Summary of metadata of newly sequenced genomes (bold type, $\mathrm{P}=$ Planomonospora). (B) Segment of autoMLST-generated phylogenetic tree.

To investigate the similarity among the Planomonospora BGCs, we processed the output of antiSMASH v5.0.0 ${ }^{8}$ with the program BiG-SCAPE/CORASON v1.0. ${ }^{46}$ This tool calculates sequence similarity networks and groups related BGCs in gene cluster families (GCFs), based on Pfam composition similarity. It further allows comparison against the MIBiG repository of experimentally established BGCs and automated calculations of phylogenetic relationships between BGCs.

The 131 BGCs from the genomes of Figure 7A could be grouped into 49 GCFs, of which 17 were singletons (only one member), as illustrated in Figure 8. Interestingly, the analysis demonstrated the existence of eight GCFs that are common among the four Planomonospora strains as well as Planobispora rosea ATCC53733. An additional GCF is present in all strains, except for ID82291, the strain with a slightly reduced genome. On the contrary, only two 
GCFs are present in the four Planomonospora genomes but not in Planobispora rosea. Only two of these eight core GCFs are highly related to experimentally established BGCs, namely those for the lantipeptide catenulipeptin and for the polyketide alkylresorcinol. Some of these GCFs are highly conserved in other genomes of members of the Streptosporangiaceae (Figure 8).

In addition to the core GCFs, strain ID67723 and Planobispora rosea ATCC53733 share six additional BGCs, consistent with the close relationship indicated in the autoMLSTgenerated phylogenetic tree. This overlap includes an erythrochelin-like BGC, as discussed later. Further, strain ID91781 and Planomonospora sphaerica JCM9374 are remarkably similar in terms of GCFs: ID91781 lacks the sphaericin BGC and another cluster of unknown function, present in Planomonospora sphaerica JCM9374, but contains instead a type I PKS BGC.

When compared to the MIBiG-repository, only 15 (30\%) of all GCFs have a matching cluster. Investigation with the program ClusterBlast showed related BGCs in nonPlanomonospora genomes. Similarities were low in the majority of cases, except for some of the core GCFs (see Figure 8).

In summary, the three analyzed genomes contain a considerable number of BGCs, with a core of BGCs present in several representatives of the Streptosporangiaceae family. Strain ID67723 appears to be very similar to Planobispora rosea ATCC53733, also in terms of BGCs. However, a larger number of genomes is needed to better understand the distribution of GCFs in Planomonospora, as demonstrated in studies on Planctomycetes ${ }^{47}$ or Salinispora. ${ }^{15,16}$ Many of the BGCs in Planomonospora remain unannotated and may encode for novel metabolites. Aiming for a better understanding of the biosynthetic capabilities, we connected genomic and metabolomic data creating a "paired-omics" dataset, as illustrated below. 


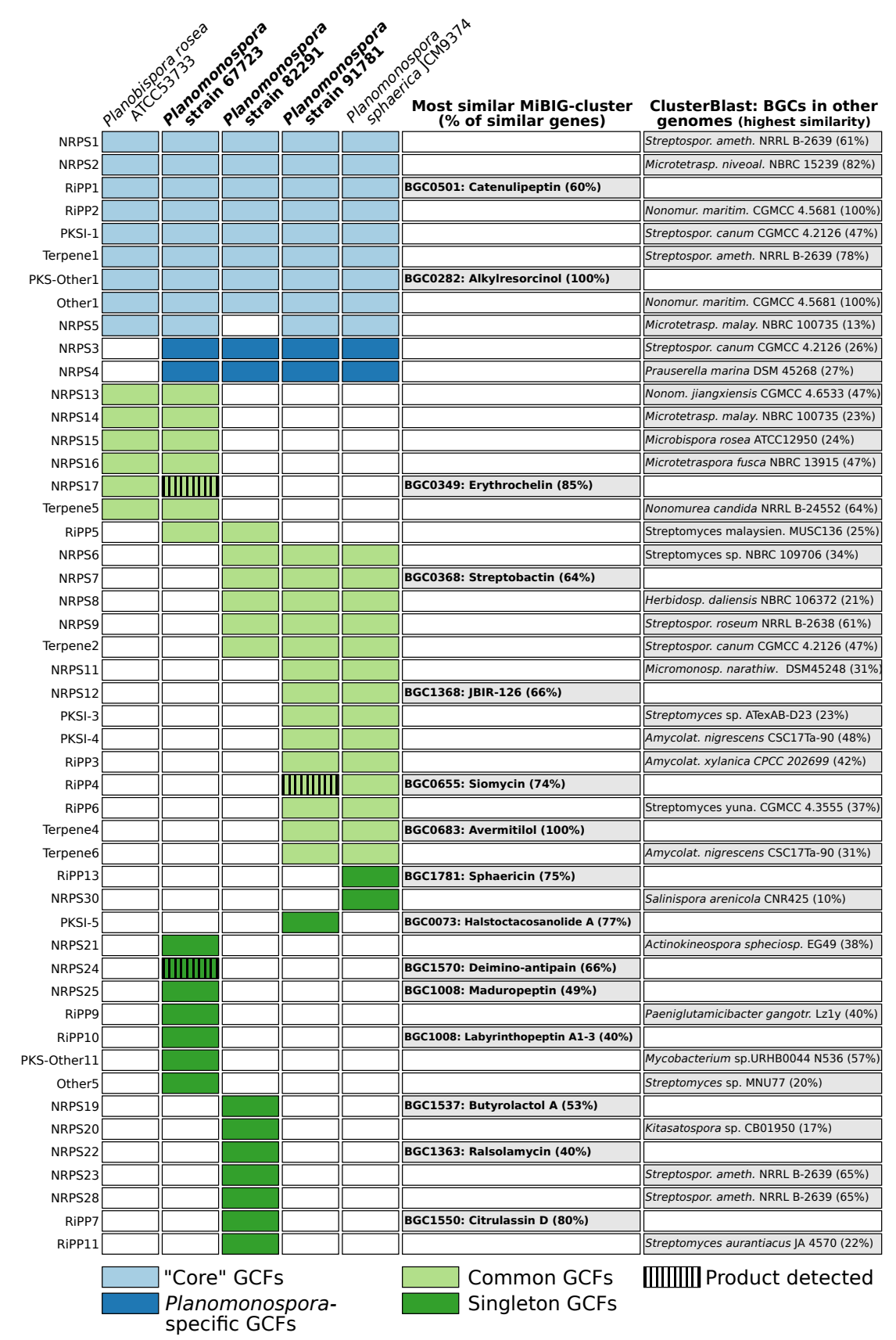

Figure 8. Distribution of biosynthetic gene clusters (BGCs) in the genomes of four Planomonospora and one Planobispora strains. Newly sequenced strains are indicated in bold. The comparison is based on similarity between genes and gene clusters, calculated by the program BiG-SCAPE. BGCs with a similarity of more than $40 \%$ to a MIBiG-deposited gene cluster were annotated. For clusters without a MIBiG-annotation, the next most similar BGC is indicated (found in public genomes by ClusterBlast). The twelve Planobispora rosea singleton BGCs were omitted, as were one BGC of strain ID67723, one of P.sphaerica and one of ID91781, fragmented due to their position on contig edges. 


\section{Paired -omics:}

Siomycin, first reported from Planomonospora in 1968 as sporangiomycin, ${ }^{23}$ is a thiostreptonlike thiopeptide with an established biosynthetic route. ${ }^{48}$ While the siomycin BGC was detected in strain ID91781 (RiPP4 in Figure 8), during metabolite annotation, it became evident that the features matching siomycin A and congeners B, C and D1 were not clustered in a single molecular family in the molecular network, as expected for structurally related molecules. Instead, they were mostly singletons. Inspection of the corresponding $\mathrm{MS}^{2}$ spectra revealed that siomycin A and congeners shared low molecular weight fragments, probably corresponding to the quinalidic acid (QA) moiety of class b thiopeptides (see Figure S6). Hence, the program MS2LDA ${ }^{49}$ was used to mine for QA-related motifs in the $\mathrm{MS}^{2}$ fragmentation spectra of features. Apart from the known siomycins, the program detected further nine putative siomycin-like thiopeptides (see Figure S7). One of them, which we named siomycin E, is hypothesized to correspond to siomycin B with one additional dehydroalanine (Dha)-residue at its C-terminal end, instead of two, as in siomycin A. $\mathrm{MS}^{2}$-fragmentation spectra of the $[\mathrm{M}+\mathrm{H}]^{+}$ions showed a particular fragment corresponding to a break between $\mathrm{Ala}^{2}$ - and $\mathrm{Dha}^{3}$ as well as $\mathrm{Thr}^{12}$ and QA, with an $m / z$-value diagnostic for each congener (see Figures 9 and S8). Literature provides a precedent for a thiopeptide with a similar intermediate molecule: thiopeptin A3a, A4a and Ba have zero, one and two Dha residues, respectively, at the C-terminal end of the molecule. ${ }^{50}$ The precursor peptide for thiopeptin, TpnA, indeed shows two serine moieties at the C-terminal end. ${ }^{51}$ Consistently, the precursor peptide encoded by the BGC RiPP4 contains two additional serine residues at the C-terminus (see Figure S9). Promiscuous processing of the C-terminal end of precursor peptides has been observed in several thiopeptides and can be also assumed here. ${ }^{48,50,52,53}$ For some of the other putative thiopeptides, the differences in exact mass with regard to siomycin A point towards one or two $N$-acetylcysteinyl-moieties with additional modifications, such as deacetylation or hydroxylation (see Figure S7). Again, literature provides precedence for likewise modified antibiotics from Streptomyces sp.: $N$-acetylcysteine derivates have been 
isolated for the macrolide piceamycin ${ }^{54}$ and the phenazine SB 212021. ${ }^{55}$ These results show how the use of additional tools such as MS2LDA can overcome the limitations of any one tool and help to organize data, identify analogues and support annotation (for a full list of annotated Mass2Motifs, see Figure S10).

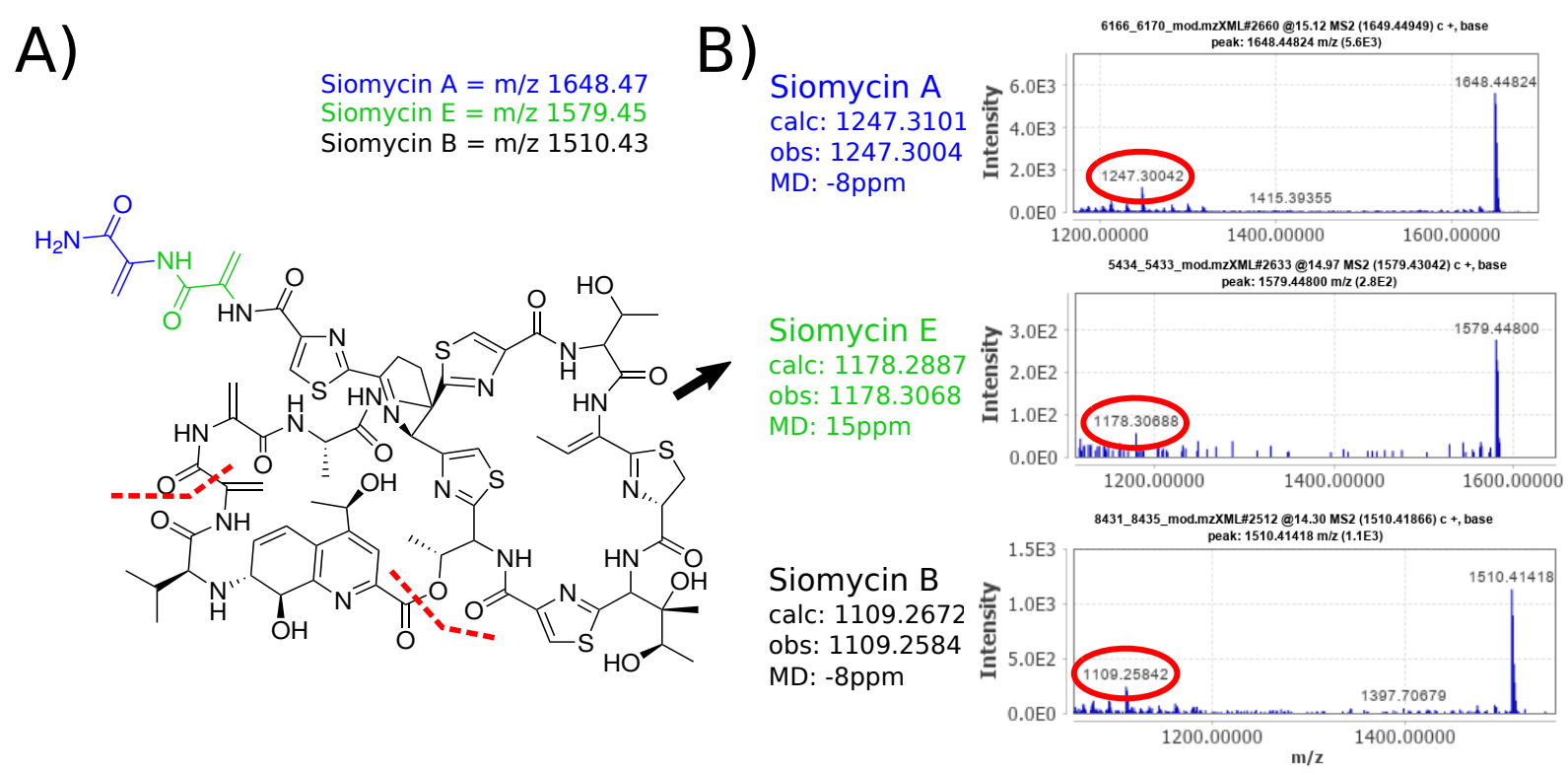

Figure 9. Identification and annotation of siomycin congeners. (A) shows the putative tandem mass fragmentation pathway of siomycins, leading to the diagnostic fragments indicated in (B). Siomycin A (blue) has two dehydroalanine (Dha)-moieties at its C-terminus end, while siomycin B (black) has none. Siomycin E (green) is hypothesized to be an intermediate congener with one Dha-group at its C-terminal end.

As mentioned above, several features were found to match desferrioxamines, iron-chelating siderophores involved in iron-uptake in bacteria, ${ }^{56,57}$ mostly in samples derived from strains of phylogroup "C", raising the question if the other Planomonospora strains produced distinctive iron-chelating molecules. In a study on 118 Salinispora genomes, Bruns et al. reported a mutually exclusive presence of either the des or slc BGC, responsible for the production of desferrioxamine or the structurally unrelated salinichelins, respectively. ${ }^{58}$ In order to rapidly identify iron-binding metabolites, we added $\mathrm{FeCl}_{3}$ to the Planomonospora extracts and reanalyzed them by LC-MS/MS, looking for mass shifts from disappearance of the iron-free form and stabilization of the iron-bound molecule, with associated Fe-characteristic isotopic 
pattern. Apart from the already identified desferrioxamines, we found that 18 features of four molecular families were also affected by the addition of iron. (see Figure 10A and Figures S12 and S14-S19). Calculation of molecular formulas and inspection of $\mathrm{MS}^{2}$-fragmentation spectra indicated relatedness between these four molecular families, but not to desferrioxamine E (see Figures S12 and S13). These four families were exclusive to 7 strains belonging to phylogroups "V2" and "S". At the same time, no desferrioxamines could be detected in extracts from these strains. Analysis with BiG-SCAPE/CORASON suggested a candidate BGC (NRPS17 in Figure 8) in one of the producer strains, ID67723. This cluster showed high similarity to the experimentally validated BGC for erythrochelin, a siderophore produced by Saccharopolyspora erythraea, as well as to a BGC with unknown function from Planobispora rosea ATCC53733, as indicated in Figure 10B. Consistently, extracts from Planobispora rosea also contained a feature with $m / z 631.3408[\mathrm{M}+\mathrm{H}]^{+}$with the calculated molecular formula $\mathrm{C}_{26} \mathrm{H}_{46} \mathrm{~N}_{8} \mathrm{O}_{10}$ (see Figure 10A). Characterisation of this metabolite confirmed a salinichelinlike structure, with a lysine instead of an arginine in position two (manuscript in preparation). For strain ID67723, 11 additional iron-shifted features were identified. The similarity of their $\mathrm{MS}^{2}$ fragmentation spectra to the features with $\mathrm{m} / z 631.3408[\mathrm{M}+\mathrm{H}]^{+}$suggests that they were also synthesized by BGC NRPS17. While many were hypothesized to be congeners differing in one or more methylene groups, feature ID314 $(\mathrm{m} / z 1067.5659)$ appears to be a glycosylated version of feature ID289 $(\mathrm{m} / z$ 905.5136).

\section{Conclusion:}

The work presented here allowed us to get unprecedented insight into the poorly explored genus Planomonospora. Among the 72 investigated strains, we found phylogenetic diversity that exceeds the (few) known isolates. Using feature-based molecular networking, we could find the majority of previously reported Planomonospora metabolites, as well as demonstrating phylogroup-specificity for many of them. We also showed that Planomonospora can 


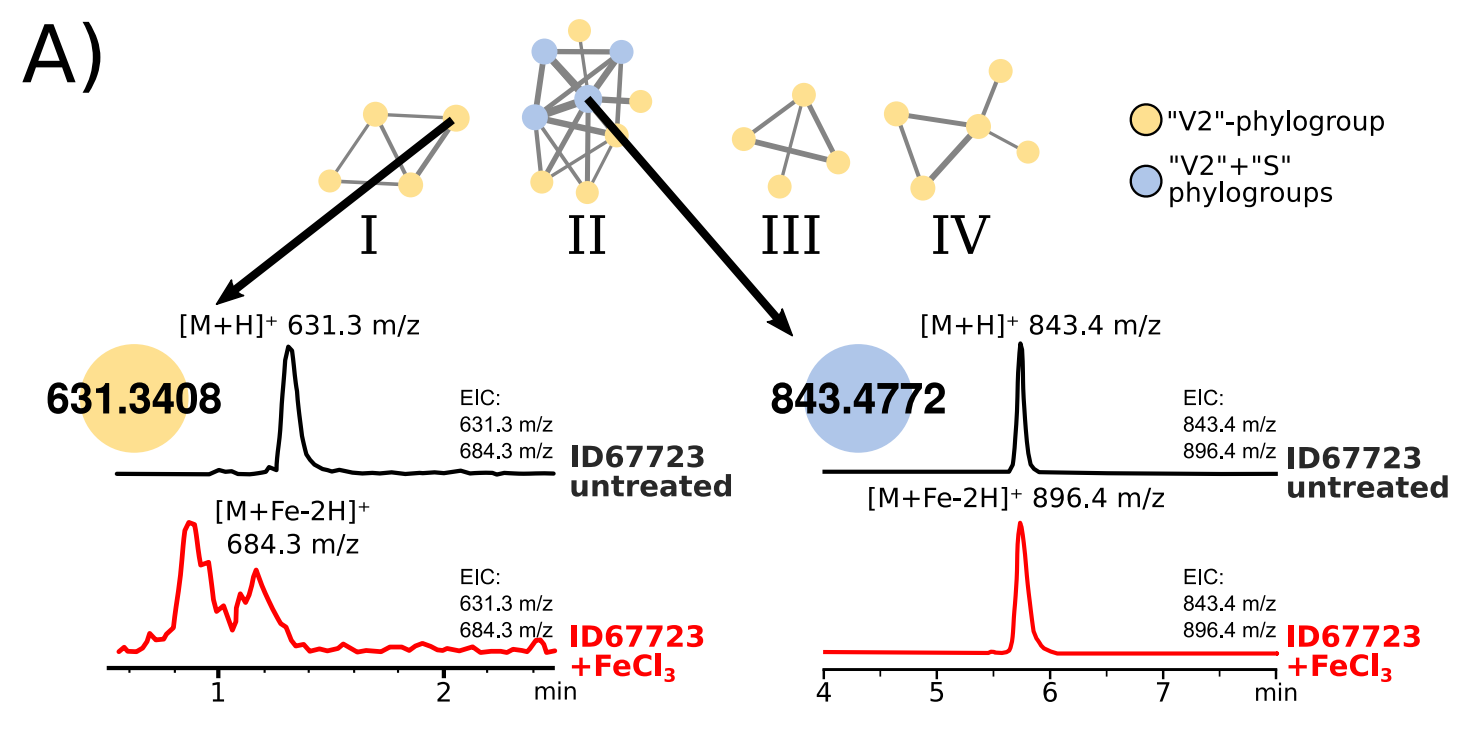

B) BGC0000349.1: Erythrochelin BGC Planomonospora sp. ID67723

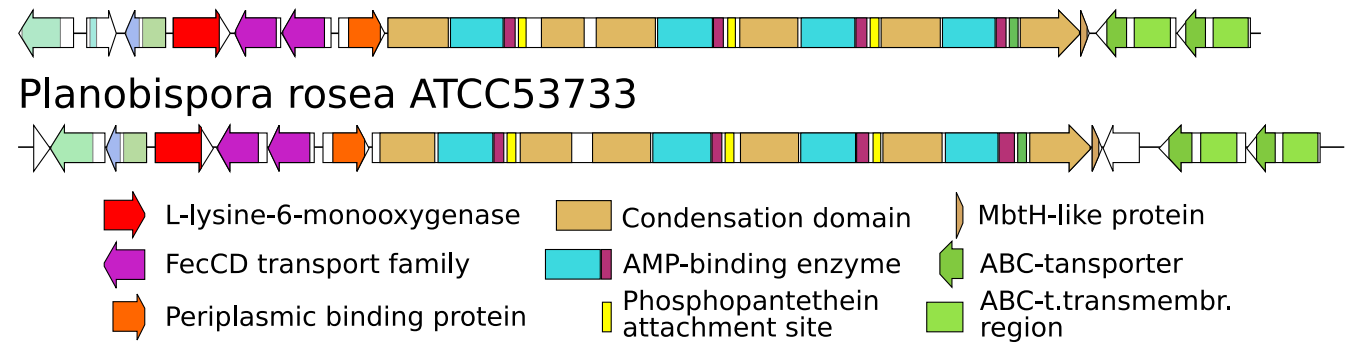

Figure 10. Investigation of some iron-binding metabolites: (A) features in several molecular families, such as $m / z 631.3$ or $m / z$ 843.4, showed iron complexion upon treatment with $\mathrm{FeCl}_{3}$. LCMS-analysis of Fe-treated samples show disappearance of the unbound form (black trace) and appearance of the Fe-bound form (red trace; for original traces, see Figure S11). (B) Comparison of the erythrochelin BGC with GCF NRPS17, shared by Planomonospora sp. ID67723 and Planobispora rosea ATCC53733.

produce desferrioxamines as iron-chelating molecules, or, alternatively, members of a different siderophore family. The majority of features detected in the Planomonospora extracts and of the BGCs of the available Planomonospora genomes remain unknown, underlying the very limited knowledge we have about this genus. 


\section{General Experimental Procedures:}

Cultivation. Strains from frozen stocks $\left(-80^{\circ} \mathrm{C}\right)$ were propagated on $\mathrm{S} 1$ plates ${ }^{59}$ at $28^{\circ} \mathrm{C}$ for two to three weeks. The grown mycelium was then homogenized with a sterile pestle and used to inoculate $15 \mathrm{~mL}$ AF (=AF/MS) ${ }^{59}$ medium in a $50 \mathrm{~mL}$ baffled flask. After cultivation on a rotary shaker $(200 \mathrm{rpm})$ at $30^{\circ} \mathrm{C}$ for 72 hours, $1.5 \mathrm{ml}$ of the exponentially growing culture was used to inoculate each $15 \mathrm{~mL}$ of MC1 (35 g/L soluble starch, $10 \mathrm{~g} / \mathrm{L}$ glucose, $2 \mathrm{~g} / \mathrm{L}$ hydrolyzed casein, $3.5 \mathrm{~g} / \mathrm{L}$ meat extract, $20 \mathrm{~g} / \mathrm{L}$ yeast extract, $10 \mathrm{~g} / \mathrm{L}$ soybean meal, $2 \mathrm{~g} / \mathrm{L} \mathrm{CaCO}_{3}$, adjusted to $\mathrm{pH} 7.2$ ), AF2 (8g/L yeast extract, $30 \mathrm{~g} / \mathrm{L}$ soybean meal, $11 \mathrm{~g} / \mathrm{L}$ glucose, $25 \mathrm{~g} / \mathrm{L}$ malt extract, $1 \mathrm{~g} / \mathrm{L}$ L-valine, 0.5g/L Biospumex (Cognis, France), adjusted to $\mathrm{pH} 7.4$ ), R3 (=RARE3) ${ }^{60}$ and AF media in a $50 \mathrm{~mL}$ baffled flask. After 7 days of cultivation as before, cultures were harvested and extracted as described below.

Extraction and LC-MS sample preparation. $10 \mathrm{~mL}$ of each culture was centrifuged at 16,000 rcf for $10 \mathrm{~min}$ and the resulting pellet separated from the supernatant. For each culture, two different extracts were prepared: one by solvent extraction of the mycelium, and one by solid-phase adsorption of the cleared broth. The mycelium was resuspended in $4 \mathrm{~mL}$ EtOH and incubated under agitation at room temperature for one hour. After centrifugation (16.000 rcf for $10 \mathrm{~min}$ ), the pellet was discarded and the mycelium extract was processed as described below. The cleared broth was stirred with $1 \mathrm{~mL}$ of HP20 resin suspension (Diaion) for one hour at room temperature. Then, the exhausted broth was discarded and the loaded HP20 resin was stirred with $4 \mathrm{~mL}$ of purified water (MilliQ, Merck), then recovered by centrifugation and eluted with $5 \mathrm{~mL}$ 80:20 MeOH: $\mathrm{H}_{2} \mathrm{O}$ under agitation at room temperature for $10 \mathrm{~min}$. Both extracts were transferred to 96 -well plates (conic bottom). For mycelium extract, $100 \mu \mathrm{L}$ of extract were deposited, while for supernatant extract, 125 $\mu \mathrm{L}$ were deposited. Extracts were dried under vacuum at $40^{\circ} \mathrm{C}$. Before analysis, extracts were rehydrated: for mycelium-extracts, $100 \mu \mathrm{L} \mathrm{90 \%} \mathrm{EtOH} \mathrm{was} \mathrm{used,} \mathrm{while} \mathrm{for} \mathrm{supernatant-}$ extracts, $125 \mu \mathrm{L} 80 \% \mathrm{MeOH}$ was utilized. The samples were centrifuged for 3 min at 16,000 
rcf to remove suspended particles.For LCMS-analysis, $50 \mu \mathrm{L}$ of mycelium extract and $50 \mu \mathrm{L}$ of supernatant extract, coming from a single culture, were combined, centrifuged for 3 min at $16.000 \mathrm{rcf}$ and transferred to HPLC vials for analysis. To test for iron-binding metabolites, $10 \mu \mathrm{L} 10 \mathrm{mg} / \mathrm{mL} \mathrm{FeCl}_{3}$ in water (MilliQ, Merck) was added to selected rehydrated extracts.

LC-MS/MS analysis. For the 286 Planomonospora extracts investigated in molecular networking, data acquisition was performed on a Dionex UltiMate 3000 (Thermo Scientific) coupled with a micrOTOF-Q III (Bruker) instrument equipped with an electrospray interface (ESI). Samples were analyzed on an Atlantis T3 $\mathrm{C}_{18} 5 \mu \mathrm{m}$ x $4.6 \mathrm{~mm}$ x $50 \mathrm{~mm}$ column maintained at $25^{\circ} \mathrm{C}$ with a flow rate of $0.3 \mathrm{~mL} / \mathrm{min}$. Phases $\mathrm{A}$ and $\mathrm{B}$ were $0.1 \%$ acetic acid in water and $0.1 \%$ acetic acid in acetonitrile (Sigma), respectively. All solvents used were LCMS-grade. A multistep program was followed that consisted of 10, 10, 100, 100, 10, $10 \%$ phase $\mathrm{B}$ at $0,1,20,26,27,30 \mathrm{~min}$, respectively. MS data were acquired in data dependent acquisition (DDA) mode over a range from 100-3000 m/z in positive ionization mode. Auto MS/MS fragmentation was achieved with rising collision energy (35-50 keV over a gradient from 500-2000 m/z) with a frequency of $4 \mathrm{~Hz}$ for all ions over a threshold of 100 . For the analysis of commercial standards and selected Planomonospora extracts with added $\mathrm{FeCl}_{3}$, LC-MS analyses were performed on a Dionex UltiMate 3000 (Thermo Scientific) coupled with an LCQ Fleet (Thermo scientific) mass spectrometer equipped with an electrospray interface (ESI) on an Atlantis T3 $\mathrm{C}_{18} 5 \mu \mathrm{m}$ x $4.6 \mathrm{~mm}$ x $50 \mathrm{~mm}$ column, as reported elsewhere. ${ }^{61}$

HR-LC-MS/MS data calibration and conversion. LCMS/MS-files, coming from the high resolution micrOTOF-Q III (Bruker) instrument, were calibrated with Bruker DataAnalysis using an internal calibrant (Na-acetate) in HPC mode, injected at the beginning of each run. The calibration was verified by inspection of medium component soyasaponin A, present in all samples (calc. $[\mathrm{M}+\mathrm{H}]^{+} 943.526 \mathrm{~m} / z$ at RT 12.7-12.9min). Average mass deviation was routinely below 15ppm. LCMS files were exported (see Figure S35) to the .mgf and .mzXML format with Bruker DataAnalysis (version 4.2 SR2 Build 
365 64bit) and further processed using an ad hoc-written Perl5 script (see Figure S35 and S36). This was necessary for further processing with MZmine2, since the export to the .mzXML format erroneously resulted in the insertion of the non-calibrated value in the precursor-entry (<precursorMz $></$ precursorMz $>$ ) of each $\mathrm{MS}^{2}$-scan, leading to high mass deviations (>100ppm) and disrupting the logical connection between $\mathrm{MS}^{1}$ and $\mathrm{MS}^{2}$ scans in the .mzXML files. .mgf files were not affected by this and thus, our script replaces the non-calibrated entries in the .mzXML file with the correct ones from the .mgf file.

Pre-processing of HR-LC-MS/MS data with MzMine2. For pre-processing with MZmine2, mzXML files were imported and subjected to the following workflow: A) MassDetection: Retention time: auto; $\mathrm{MS}^{1}$ noise level: $1 \mathrm{E} 3 ; \mathrm{MS}^{2}$ noise level: 2E1. B) ADAP chromatogram builder: ${ }^{62}$ Retention time: auto; MS-level: 1; Min group size in \# of scans: 8; Group intensity threshold: 5E2; Min highest intensity: 1E3; $\mathrm{m} / z$ tolerance: 20ppm; C) Chromatogram deconvolution: Baseline-cutoff-algorithm; Min peak height: 1E3; Peak duration: 0.1-1.3 min; Baseline level: 2.5E2; $\mathrm{m} / z$ range $\mathrm{MS}^{2}$ pairing: 0.02 ; RT range $\mathrm{MS}^{2}$ pairing: $0.4 \mathrm{~min}$; D) Isotopic peaks grouper: $\mathrm{m} / \mathrm{z}$ tolerance: $20 \mathrm{ppm}$; RT tolerance: 0.2 min; Monotonic shape: no; Maximum charge: 2; Representative isotope: Most intense; E) RANSAC peak alignment: $m / z$ tolerance: 20 ppm; RT tolerance: 0.7 min; RT tolerance after correction: $0.35 \mathrm{~min}$; RANSAC iterations: 100000; Minimum number of points: 50\%; Threshold value: 0.5; Linear model: no; Require same charge state: no; F) Duplicate peak filter: Filter mode: New average; $\mathrm{m} / \mathrm{z}$ tolerance: $0.02 \mathrm{~m} / z$; RT tolerance: $0.4 \mathrm{~min}$. Features with no accompanying $\mathrm{MS}^{2}$ data were excluded from the analysis. Features present in both samples and media blanks were excluded as well, since they related to media components. Features with $m / z$-values of $<300$ or a retention time $<1.5$ min or $>20$ min were also excluded. The resulting feature list contained 1492 entries and was exported to the GNPS-compatible format. 


\section{Global Natural Products Social Molecular Networking (GNPS) feature-based} molecular MS/MS network. Using the Feature-Based Molecular Networking (FBMN) workflow (version release_14) ${ }^{35}$ on GNPS, ${ }^{12}$ a molecular network was created by processing the output of MZmine2. Parameters were adapted from the GNPS documentation: $\mathrm{MS}^{2}$ spectra were filtered so that all MS/MS fragment ions within +/- 17 Da of the precursor $m / z$ were removed and only the top 6 fragment ions in the +/- 50 Da window through the spectrum were utilized, with a minimum fragment ions intensity of 50. Both the MS/MS fragment ion tolerance and the precursor ion mass tolerance were set to 0.03 Da. Edges of the created molecular network were filtered to have a cosine score above 0.7 and more that 5 matched peaks between the connected nodes. Further, these were only kept in the network if each of the nodes appeared in each other's respective top 10 most similar nodes. The maximum size of molecular families in the network was set to 250 and the lowest scoring edges from each family were removed until member count was below this threshold. The $\mathrm{MS}^{2}$ spectra in the molecular network were searched against GNPS spectral libraries. ${ }^{12,63}$ Reported matches between network and library spectra were required to have a score above 0.6 and at least 5 matched peaks. The DEREPLICATOR-program was used to annotate MS/MS spectra. ${ }^{64}$ The molecular networks were visualized using Cytoscape 3.7.1. ${ }^{36}$ The molecular networking job is accessible by the link https://gnps.ucsd.edu/ProteoSAFe/status.jsp?task=92036537c21b44c29e509291e53f6382. HR-ESI-LC-MS/MS data were deposited in MassIVE (MSV000085376) and linked with the genomic data at the iOMEGA Pairing Omics Data Platform (http://pairedomicsdata.bioinformatics.nl/projects).

MS2LDA analysis. The molecular networking job described above was analyzed by MS2LDA (version release_14), accessing the tool directly on the GNPS website. Parameters were set as follows: Bin Width: 0.01; Nr of LDA iterations: 1000; Min MS2 Intensity: 100; LDA Free Motifs: 500. All MotifDBs except "Streptomyces and Salinisporus Motif In- 
clusion" were excluded. Further parameters were left at default (Overlap score threshold: 0.3; Probability value threshold: 0.1; TopX in node: 5). Results were uploaded to the MS2LDAwebsite, with the width of ms2 bins set to $0.005 \mathrm{Da}$, as recommended (http://ms2lda.org/).

16S rRNA gene analysis. For 16S rRNA gene amplification, single colonies were picked from S1 medium plates and lysed at $95^{\circ} \mathrm{C}$ in $100 \mu \mathrm{L}$ PCR-grade water for $5 \mathrm{~min} .5 \mu \mathrm{L}$ centrifuged lysate was added to the reaction mix, containing $25 \mu \mathrm{L}$ DreamTaq Green PCR Master Mix 2X (Thermo Scientific), $3 \mu \mathrm{L}$ of $10 \mathrm{x}$ Denhardt's reagents, ${ }^{65}$ each $500 \mathrm{nM}$ of eubacterial primers R1492 and F27 $7^{66}$ and $12 \mu \mathrm{L}$ water, resulting in a final volume of $50 \mu \mathrm{L}$. The amplification was performed as reported elsewhere. ${ }^{66}$ PCR products were sequenced using Sanger sequencing by an external DNA sequencing provider (Cogentech, Milan, IT), with the primers mentioned above. 16S rRNA gene sequences were inspected and assembled manually using the software AliView, ${ }^{67}$ yielding 35 non-redundant sequences with a consensus length of $1377 \mathrm{bp}$. The sequences were analyzed with programs contained in the PHYLIP package ${ }^{68,69}$ as reported elsewhere, ${ }^{66}$ with slight modifications (bootstrapped with 1000 replicates). The resulting consensus tree was visualized using the iTOL-webserver. ${ }^{70,71}$ Sequences were deposited in GeneBank, with accession numbers included in the supplemental information (Figures S1-2) of this study.

Isolation of gDNA. To isolate genomic DNA (gDNA), mycelium from $5 \mathrm{~mL}$ Planomonospora cultures, cultivated in AF medium for 72 hours as described above, was extracted with standard protocols for Streptomyces by phenol-chloroform, as described elsewhere. ${ }^{44}$ gDNA was sequenced with both Illumina and PacBio technologies by an external service provider (Macrogen, Seoul, KOR) and assembled using the program SPAdes (3.11). Genome sequences were deposited in GenBank under the BioProject ID PRJNA633779, with accession numbers JABTEX000000000 (ID82291), JABTEY000000000 (ID91781) and JABTEZ000000000 (ID67723). 
BiG-SCAPE sequence similarity network. .gbk-files of BGCs detected by the webbased application antiSMASH 5.0 (https://antismash.secondarymetabolites.org/) were analyzed including the ClusterFinder algorithm and compared to MIBiG-deposited BGCs using BiG-SCAPE 1.0 in "hybrids" mode. All setting were left to default, except the cutoff-value, which was set to 0.5 .

Phylogenetic multilocus sequence analysis (autoMLST). The web-based application autoMLST (http://automlst.ziemertlab.com/analyze\#) was used to perform a multilocus sequence analysis in "denovo mode" and default settings.

\section{ACKNOWLEDGEMENT}

The authors thank Christian Milani and Marco Ventura for genome reassembly and data curation, Paolo Monciardini for suggestions regarding the phylogenetic analysis and the whole Naicons team for helpful comments and discussion. M.M.Z. thanks Prof. Tanneke den Blaauwen for guidance and advice. This work has received funding from the European Union's Horizon 2020 research and innovation program under grant agreement No.721484 (Train2Target). M.C. received funding from the German Research Foundation (DFG) grant nr. FOR2372.

\section{ASSOCIATED CONTENT}

The following files are available free of charge.

- Supporting Information: Figures S1-S38. 


\section{REFERENCES}

(1) Newman, D. J.; Cragg, G. M. Natural products as sources of new drugs over the nearly four decades from 01/1981 to 09/2019. J. Nat. Prod. 2020, 83, 770-803.

(2) Ortholand, J.-Y.; Ganesan, A. Natural products and combinatorial chemistry: back to the future. Curr. Opin. Chem. Biol. 2004, 8, 271-280.

(3) Selva, E. Growing the seeds sown by Piero Sensi. J. Antibiot. 2014, 67, 613.

(4) da Silva, R. R.; Dorrestein, P. C.; Quinn, R. A. Illuminating the dark matter in metabolomics. Proc. Natl. Acad. Sci. U. S. A. 2015, 112, 12549-12550.

(5) Wolfender, J.-L.; Litaudon, M.; Touboul, D.; Queiroz, E. F. Innovative omics-based approaches for prioritisation and targeted isolation of natural products-new strategies for drug discovery. Nat. Prod. Rep. 2019, 36, 855-868.

(6) Yera, E. R.; Cleves, A. E.; Jain, A. N. Chemical structural novelty: on-targets and off-targets. J. Med. Chem. 2011, 54, 6771-6785.

(7) Cimermancic, P.; Medema, M. H.; Claesen, J.; Kurita, K.; Brown, L. C. W.; Mavrommatis, K.; Pati, A.; Godfrey, P. A.; Koehrsen, M.; Clardy, J.; Birren, B.; Takano, E.; Sali, A.; Linington, R.; Fischbach, M. Insights into secondary metabolism from a global analysis of prokaryotic biosynthetic gene clusters. Cell 2014, 158, 412-421.

(8) Blin, K. et al. antiSMASH 4.0 - improvements in chemistry prediction and gene cluster boundary identification. Nucleic Acids Res. 2017, 45, W36-W41.

(9) Kautsar, S. A. et al. MIBiG 2.0: a repository for biosynthetic gene clusters of known function. Nucleic Acids Res. 2020, 48, D454-D458.

(10) Yang, J. Y. et al. Molecular networking as a dereplication strategy. J. Nat. Prod. 2013, 76, 1686-1699. 
(11) Ramos, A. E. F.; Evanno, L.; Poupon, E.; Champy, P.; Beniddir, M. A. Natural products targeting strategies involving molecular networking: different manners, one goal. Nat. Prod. Rep. 2019, 36, 960-980.

(12) Wang, M. et al. Sharing and community curation of mass spectrometry data with Global Natural Products Social Molecular Networking. Nat. Biotechnol. 2016, 34, 828.

(13) van Santen, J. A. et al. The natural products atlas: an open access knowledge base for microbial natural products discovery. ACS Cent. Sci. 2019, 5, 1824-1833.

(14) Crusemann, M.; ONeill, E. C.; Larson, C. B.; Melnik, A. V.; Floros, D. J.; da Silva, R. R.; Jensen, P. R.; Dorrestein, P. C.; Moore, B. S. Prioritizing natural product diversity in a collection of 146 bacterial strains based on growth and extraction protocols. J. Nat. Prod. 2016, 80, 588-597.

(15) Duncan, K. R.; Crüsemann, M.; Lechner, A.; Sarkar, A.; Li, J.; Ziemert, N.; Wang, M.; Bandeira, N.; Moore, B. S.; Dorrestein, P. C.; Jensen, P. Molecular networking and pattern-based genome mining improves discovery of biosynthetic gene clusters and their products from Salinispora species. Chem. Biol. 2015, 22, 460-471.

(16) Ziemert, N.; Lechner, A.; Wietz, M.; Millán-Aguiñaga, N.; Chavarria, K. L.; Jensen, P. R. Diversity and evolution of secondary metabolism in the marine actinomycete genus Salinispora. Proc. Natl. Acad. Sci. U. S. A. 2014, 111, E1130-E1139.

(17) Männle, D.; McKinnie, S. M.; Mantri, S. S.; Steinke, K.; Lu, Z.; Moore, B. S.; Ziemert, N.; Kaysser, L. Comparative genomics and metabolomics in the genus Nocardia. Msystems 2020, 5 .

(18) Krug, D.; Zurek, G.; Revermann, O.; Vos, M.; Velicer, G. J.; Müller, R. Discovering the hidden secondary metabolome of Myxococcus xanthus: a study of intraspecific diversity. Appl. Environ. Microbiol. 2008, 74, 3058-3068. 
(19) Maansson, M.; Vynne, N. G.; Klitgaard, A.; Nybo, J. L.; Melchiorsen, J.; Nguyen, D. D.; Sanchez, L. M.; Ziemert, N.; Dorrestein, P. C.; Andersen, M. R.; Gram, L. An integrated metabolomic and genomic mining workflow to uncover the biosynthetic potential of bacteria. Msystems 2016, 1 .

(20) Monciardini, P.; Iorio, M.; Maffioli, S.; Sosio, M.; Donadio, S. Discovering new bioactive molecules from microbial sources. Microb. Biotechnol. 2014, 7, 209-220.

(21) Thiemann, J. E. A new genus of the Actinoplanaceae: Planomonospora gen. nov. G. Microbiology 1967, 15, 27-38.

(22) Mertz, F. P. Planomonospora alba sp. nov. and Planomonospora sphaerica sp. nov., two new species isolated from soil by baiting techniques. Int. J. Syst. Evol. Microbiol. $1994,44,274-281$.

(23) Thiemann, J.; Coronelli, C.; Pagani, H.; Beretta, G.; Tamoni, G.; Arioli, V. Sporangiomycin, an antibacterial agent isolated from Planomonospora parontospora var. antibiotica var. nov. J. Antibiot. 1968, 21, 525-531.

(24) Ebata, M.; Miyazaki, K.; Otsuka, H. Studies on siomycin. I. J. Antibiot. 1969, 22, 364-368.

(25) Maffioli, S. I.; Potenza, D.; Vasile, F.; De Matteo, M.; Sosio, M.; Marsiglia, B.; Rizzo, V.; Scolastico, C.; Donadio, S. Structure revision of the lantibiotic 97518. J. Nat. Prod. 2009, 72, 605-607.

(26) Castiglione, F.; Cavaletti, L.; Losi, D.; Lazzarini, A.; Carrano, L.; Feroggio, M.; Ciciliato, I.; Corti, E.; Candiani, G.; Marinelli, F. A novel lantibiotic acting on bacterial cell wall synthesis produced by the uncommon actinomycete Planomonospora sp. Biochemistry 2007, 46, 5884-5895. 
(27) Maffioli, S. I.; Monciardini, P.; Catacchio, B.; Mazzetti, C.; Munch, D.; Brunati, C.; Sahl, H.-G.; Donadio, S. Family of class I lantibiotics from actinomycetes and improvement of their antibacterial activities. ACS Chem. Biol. 2015, 10, 1034-1042.

(28) Kodani, S.; Inoue, Y.; Suzuki, M.; Dohra, H.; Suzuki, T.; Hemmi, H.; OhnishiKameyama, M. Sphaericin, a lasso peptide from the rare actinomycete Planomonospora sphaerica. Eur. J. Org. Chem. 2017, 2017, 1177-1183.

(29) Wingender, W.; von Hugo, H.; Frommer, W.; Schäfer, D. A protease inhibitor isolated from Planomonospora parontospora. J. Antibiot. 1975, 28, 611-612.

(30) Suda, H.; Aoyagi, T.; Hamada, M.; Takeuchi, T.; Umezawa, H. Antipain, a new protease inhibitor isolated from actinomycetes. J. Antibiot. 1972, 25, 263-265.

(31) Suriyachadkun, C.; Ngaemthao, W.; Chunhametha, S. Planomonospora corallina sp. nov., isolated from soil. Int. J. Syst. Evol. Microbiol. 2016, 66, 3224-3229.

(32) Chaouch, F. C.; Bouras, N.; Mokrane, S.; Bouznada, K.; Zitouni, A.; Pötter, G.; Spröer, C.; Klenk, H.-P.; Sabaou, N. Planomonospora algeriensis sp. nov., an actinobacterium isolated from a Saharan soil of Algeria. Antonie van leeuwenhoek 2017, $110,245-252$.

(33) Pluskal, T.; Castillo, S.; Villar-Briones, A.; Orešič, M. MZmine 2: modular framework for processing, visualizing, and analyzing mass spectrometry-based molecular profile data. BMC Bioinf. 2010, 11, 395.

(34) Olivon, F.; Gwendal, G.; Roussi, F.; Litaudon, M.; Touboul, D. MZmine 2 data-preprocessing to enhance molecular networking reliability. Anal. Chem. 2017, 78367840.

(35) Nothias, L. F. et al. Feature-based molecular networking in the GNPS analysis environment. bioRxiv 2019, 
(36) Shannon, P.; Markiel, A.; Ozier, O.; Baliga, N. S.; Wang, J. T.; Ramage, D.; Amin, N.; Schwikowski, B.; Ideker, T. Cytoscape: a software environment for integrated models of biomolecular interaction networks. Genome Res. 2003, 13, 2498-2504.

(37) Hoffmann, T.; Krug, D.; Bozkurt, N.; Duddela, S.; Jansen, R.; Garcia, R.; Gerth, K.; Steinmetz, H.; Müller, R. Correlating chemical diversity with taxonomic distance for discovery of natural products in myxobacteria. Nat. Commun. 2018, 9, 1-10.

(38) Hubert, J.; Nuzillard, J.-M.; Renault, J.-H. Dereplication strategies in natural product research: How many tools and methodologies behind the same concept? Phytochem. Rev. 2017, 16, 55-95.

(39) Beutler, J. A.; Alvarado, A. B.; Schaufelberger, D. E.; Andrews, P.; McCloud, T. G. Dereplication of phorbol bioactives: Lyngbya majuscula and Croton cuneatus. J. Nat. Prod. 1990, 53, 867-874.

(40) Sumner, L. W. et al. Proposed minimum reporting standards for chemical analysis. Metabolomics 2007, 3, 211-221.

(41) Imbert, M.; Béchet, M.; Blondeau, R. Comparison of the main siderophores produced by some species of Streptomyces. Curr. Microbiol. 1995, 31, 129-133.

(42) Dohra, H.; Suzuki, T.; Inoue, Y.; Kodani, S. Draft genome sequence of Planomonospora sphaerica JCM9374, a rare actinomycete. Genome Announc. 2016, 4, e00779-16.

(43) Zdouc, M. M.; Alanjary, M. M.; Maffioli, S. I.; Crüsemann, M.; Medema, M. M.; Donadio, S.; Sosio, M. A biaryl-linked tripeptide from Planomonospora leads to discovery of widespread class of minimal RiPP gene clusters. bioRxiv 2020,

(44) Tocchetti, A.; Bordoni, R.; Gallo, G.; Petiti, L.; Corti, G.; Alt, S.; Cruz, J. C.; Salzano, A. M.; Scaloni, A.; Puglia, A. M.; De Bellis, G.; Peano, C.; Donadio, S.; 
Sosio, M. A genomic, transcriptomic and proteomic look at the GE2270 producer Planobispora rosea, an uncommon actinomycete. PLOS ONE 2015, 10.

(45) Alanjary, M.; Steinke, K.; Ziemert, N. AutoMLST: an automated web server for generating multi-locus species trees highlighting natural product potential. Nucleic Acids Res. 2019, 47, W276-W282.

(46) Navarro-Muñoz, J. C. et al. A computational framework to explore large-scale biosynthetic diversity. Nat. Chem. Biol. 2020, 16, 60-68.

(47) Wiegand, S. et al. Cultivation and functional characterization of 79 Planctomycetes uncovers their unique biology. Nat. Microbiol. 2020, 5, 126-140.

(48) Liao, R.; Duan, L.; Lei, C.; Pan, H.; Ding, Y.; Zhang, Q.; Chen, D.; Shen, B.; Yu, Y.; Liu, W. Thiopeptide biosynthesis featuring ribosomally synthesized precursor peptides and conserved posttranslational modifications. Chem. Biol. 2009, 16, 141-147.

(49) Wandy, J.; Zhu, Y.; van der Hooft, J. J.; Daly, R.; Barrett, M. P.; Rogers, S. Ms2lda.org: web-based topic modelling for substructure discovery in mass spectrometry. Bioinformatics 2017, 34, 317-318.

(50) Duan, L.; Wang, S.; Liao, R.; Liu, W. Insights into quinaldic acid moiety formation in thiostrepton biosynthesis facilitating fluorinated thiopeptide generation. Chem. Biol. 2012, 19, 443-448.

(51) Ichikawa, H.; Bashiri, G.; Kelly, W. L. Biosynthesis of the thiopeptins and identification of an F420H2-dependent dehydropiperidine reductase. J. Am. Chem. Soc. 2018, 140, 10749-10756.

(52) Just-Baringo, X.; Albericio, F.; Alvarez, M. Thiopeptide engineering: A multidisciplinary effort towards future drugs. Angew. Chem., Int. Ed. 2014, 53, 6602-6616. 
(53) Zhang, Q.; Liu, W. Biosynthesis of thiopeptide antibiotics and their pathway engineering. Nat. Prod. Rep. 2013, 30, 218-226.

(54) Schulz, D.; Nachtigall, J.; Riedlinger, J.; Schneider, K.; Poralla, K.; Imhoff, J. F.; Beil, W.; Nicholson, G.; Fiedler, H.-P.; Süssmuth, R. D. Piceamycin and its Nacetylcysteine adduct is produced by Streptomyces sp. GB 4-2. J. Antibiot. 2009, 62, $513-518$.

(55) Gilpin, M. L.; Fulston, M.; Payne, D.; Cramp, R.; Hood, I. Isolation and structure determination of two novel phenazines from a Streptomyces with inhibitory activity against metallo-enzymes, including metallo-b-lactamase. J. Antibiot. 1995, 48, 10811085.

(56) Keberle, H. The biochemistry of desferrioxamine and its relation to iron metabolism. Ann. N. Y. Acad. Sci. 1964, 119, 758-768.

(57) Winkelmann, G. Microbial siderophore-mediated transport. Biochem. Soc. Trans. 2002, 30, 691-696.

(58) Bruns, H.; Crüsemann, M.; Letzel, A.-C.; Alanjary, M.; McInerney, J. O.; Jensen, P. R.; Schulz, S.; Moore, B. S.; Ziemert, N. Function-related replacement of bacterial siderophore pathways. ISME J 2018, 12, 320-329.

(59) Donadio, S.; Monciardini, P.; Sosio, M. Approaches to discovering novel antibacterial and antifungal agents. Methods Enzymol. 2009, 458, 3-28.

(60) Sosio, M.; Stinchi, S.; Beltrametti, F.; Lazzarini, A.; Donadio, S. The gene cluster for the biosynthesis of the glycopeptide antibiotic A40926 by Nonomuraea species. Chem. Biol. 2003, 10, 541-549.

(61) Iorio, M.; Tocchetti, A.; Cruz, J.; Del Gatto, G.; Brunati, C.; Maffioli, S.; Sosio, M.; 
Donadio, S. Novel polyethers from screening Actinoallomurus spp. Antibiotics 2018, 7, 47.

(62) Myers, O. D.; Sumner, S. J.; Li, S.; Barnes, S.; Du, X. One step forward for reducing false positive and false negative compound identifications from mass spectrometry metabolomics data: new algorithms for constructing extracted ion chromatograms and detecting chromatographic peaks. Anal. Chem. 2017, 89, 8696-8703.

(63) Horai, H. et al. MassBank: a public repository for sharing mass spectral data for life sciences. J. Mass Spectrom. 2010, 45, 703-714.

(64) Mohimani, H.; Gurevich, A.; Shlemov, A.; Mikheenko, A.; Korobeynikov, A.; Cao, L.; Shcherbin, E.; Nothias, L.-F.; Dorrestein, P. C.; Pevzner, P. A. Dereplication of microbial metabolites through database search of mass spectra. Nat. Commun. 2018, 9, 4035.

(65) Volossiouk, T.; Robb, E. J.; Nazar, R. N. Direct DNA extraction for PCR-mediated assays of soil organisms. Appl. Environ. Microbiol. 1995, 61, 3972-3976.

(66) Monciardini, P.; Sosio, M.; Cavaletti, L.; Chiocchini, C.; Donadio, S. New PCR primers for the selective amplification of $16 \mathrm{~S}$ rDNA from different groups of actinomycetes. FEMS Microbiol. Ecol. 2002, 42, 419-429.

(67) Larsson, A. AliView: a fast and lightweight alignment viewer and editor for large datasets. Bioinformatics 2014, 30, 3276-3278.

(68) Felsenstein, J. PHYLIP (phylogeny inference package), version 3.5 c; Joseph Felsenstein., 1993.

(69) PHYLIP. http://evolution.genetics.washington.edu/phylip.html, Accessed: $06 / 11 / 2019$. 
bioRxiv preprint doi: https://doi.org/10.1101/2020.07.19.210815; this version posted July 19, 2020. The copyright holder for this preprint (which

was not certified by peer review) is the author/funder, who has granted bioRxiv a license to display the preprint in perpetuity. It is made available under aCC-BY-NC-ND 4.0 International license.

(70) Letunic, I.; Bork, P. Interactive Tree Of Life (iTOL) v4: recent updates and new developments. Nucleic Acids Res. 2019,

(71) iTOL Webserver. https://itol.embl.de/, Accessed: 06/11/2019. 